\title{
Erratum
}

\section{Renormalization effects in the superstring moduli from the higher-derivative terms}

\author{
[Int. J. Mod. Phys. A, Vol. 16, No. 19, 3217-3235 (2001)] \\ M. D. Pollock* \\ V. A. Steklov Mathematical Institute, Russian Academy of Sciences, \\ Ulitsa Gubkina 8, Moscow 119991, Russia* \\ *mdp30@cam.ac.uk
}

Received 11 March 2016

Published 28 March 2016

The following are corrections to errors in the published paper:

(1) On p. 3217, in line 30, and on p. 3220, in line 15, printed as " $1.14<\gamma<2.63$ " should read " $1.23<\gamma<2.26$ "

(2) On p. 3228, in line 8, printed as

$$
+\left\{N-\left[2(N+1) \hat{\alpha}_{1}-8 \hat{\alpha}_{2}\right] e^{-2 \alpha} R\right\}(\nabla \beta)^{2}
$$

should read

$$
+\left\{N-\left[2 N(N+1) \hat{\alpha}_{1}-8 \hat{\alpha}_{2}\right] e^{-2 \alpha} R\right\}(\nabla \beta)^{2}
$$

(3) On p. 3229, in line 3, printed as

$$
+3\left(1-\frac{23 \alpha^{\prime}}{24} e^{-2 \alpha} R\right) \frac{(\nabla \beta)^{2}}{\kappa^{2}}
$$

should read

$$
+3\left(1-\frac{29 \alpha^{\prime}}{12} e^{-2 \alpha} R\right) \frac{(\nabla \beta)^{2}}{\kappa^{2}}
$$

*Temporary address. 
(4) On p. 3230, lines 10, 16 should read, respectively,

$$
\begin{aligned}
& b=\kappa^{-2}\left\{\frac{1}{2} N+\left\{\left(N \hat{\alpha}_{2}+8 \hat{\alpha}_{3}\right) f-\left[N(N+1) \hat{\alpha}_{1}-4 \hat{\alpha}_{2}\right] g\right\} \kappa^{2} \rho e^{-2 \alpha}\right\}, \\
& b=\kappa^{-2}\left[3-(99 \gamma-124) \kappa^{4} \rho e^{-2 \alpha}\right],
\end{aligned}
$$

(5) On p. 3231, lines 2, 3 should read

$$
\begin{aligned}
& \mathcal{A}=2(15 \gamma-14)^{2}+30(2-\gamma)(99 \gamma-124), \\
& \mathcal{B}=-90(2-\gamma)+(198 \gamma-248),
\end{aligned}
$$

(6) On p. 3231, Eq. (77) should read

$$
Z_{ \pm}=\frac{107-72 \gamma \pm \sqrt{(107-72 \gamma)^{2}-6\left(630 \gamma^{2}-2,205 \gamma+1,762\right)}}{-1,260 \gamma^{2}+4,410 \gamma-3,524}
$$

(7) On p. 3231, lines 9, 10 should read that is throughout the range

$$
1.23<\gamma<2.26
$$

(8) On p. 3231, in line 12, printed as "1.14" should read "1.23"

(9) On p. 3232, Table 1 should read

Table 1

\begin{tabular}{cccc}
\hline$\gamma$ & $4 / 3$ & $5 / 3$ & 2 \\
\hline$Z_{+}$ & 0.28 & 0.064 & 0.036 \\
$t_{c} / t_{\mathrm{P}}$ & 12 & 18 & 21 \\
$S_{\mathrm{h}}$ & 60 & 31 & 43 \\
\hline
\end{tabular}

